\title{
Spectral Transformations for Two-Dimensional Filters via FFT
}

\author{
Nikos E. Mastorakis, Senior Member, IEEE, and M. N. S. Swamy, Life Fellow, IEEE
}

\begin{abstract}
In this paper, a new fast algorithm for spectral transformations for two-dimensional digital filters is presented. The algorithm is based on the use of the fast Fourier transform. The computational complexity of this algorithm is evaluated. The simplicity and efficiency of the algorithm is illustrated by a numerical example.
\end{abstract}

Index Terms-Discrete transforms, fast Fourier transforms, spectral transformation, two-dimensional filters.

\section{INTRODUCTION}

$\mathbf{T}$ WO-DIMENSIONAL (2-D) digital filters have found numerous applications within the vast context of 2-D digital signal processing. In the area of real-time image processing, for example, they are used for the enhancement of X-ray images, blood cell analysis, thermography, ultrasound echography, computerized tomography, moving-objects recognition, underwater acoustics, remote-sensing, and robotics. Several implementation algorithms for 2-D digital filters have been widely reported [1]-[3]. Most of these algorithms are based on proper (2-D) rational (also called spectral) transformations. These transformations are useful for designing low-pass, high-pass, band-pass, and multiple passband filters. The bilinear transformation is a special case of the 1-D rational transformation. This transformation appears to have a long list of references in the recent literature [5]-[10].

It is well known that a linear shift-invariant causal singleinput single-output (SISO) 2-D system can be described by the transfer function

$$
G\left(s_{1}, s_{2}\right)=\frac{Q\left(s_{1}, s_{2}\right)}{F\left(s_{1}, s_{2}\right)}
$$

where $Q\left(s_{1}, s_{2}\right)$ and $F\left(s_{1}, s_{2}\right)$ are coprime polynomials in the independent complex variables $s_{1}$ and $s_{2}$. As a unifying approach, the variables $s_{1}$ and $s_{2}$ are used for the continuous as well as for the discrete 2-D case. A transformation $T$ is a mapping: $\mathbf{C}^{2} \rightarrow \mathbf{C}^{2}$ with $s_{1}=G_{1}\left(z_{1}, z_{2}\right), s_{2}=G_{2}\left(z_{1}, z_{2}\right)$. Obviously the problem of transformation of the rational function (1) is reduced to transforming the polynomials $Q\left(s_{1}, s_{2}\right)$ and $F\left(s_{1}, s_{2}\right)$. Henceforth, we deal only with the 2-D rational transformation of the 2-D polynomial $F\left(s_{1}, s_{2}\right)$.

Manuscript received July 16, 2001; revised January 17, 2002. This paper was recommended by Co-Guest Editor S. Basu.

N. E. Mastorakis is with the Department of Computer Science, Hellenic Nava Academy, Military Institutions of University Education, Piraeus 18539, Greece (e-mail: mastor@ieee.org).

M. N. S. Swamy is with the Department of Electrical and Computer Engineering, Concordia University, Montreal H3G 1M8, Canada (e-mail swamy@ece.concordia.ca).

Publisher Item Identifier S 1057-7122(02)05599-X.
A good many efficient techniques and fast algorithms for 1-D polynomial, and 1-D rational transformations of 1-D and $m$-D polynomials have been proposed lately. In [4]-[13], the bilinear and related transformations are carried out by using appropriate matrix multiplications. In [14], the same transformations are used in the multidimensional systems stability. In [15], a procedure for transforming a rational function to another rational function under an arbitrary rational transformation via appropriate matrix multiplication is outlined. In [16], the matrix multiplication technique for bilinear transformation of multivariable polynomials is developed as an extension of the corresponding 1-D technique. In [17], the same technique is applied for the discrete system stability. In [18], an efficient algorithm for bilinear transformation of multivariable polynomials is given. In [19], the bilinear transformation is extended to transform multivariable polynomials, using discrete convolution and the Kronecker product. In [20], some properties of the matrix associated with the bilinear transformation are given. In [21], properties of the same matrix in the multidimensional case are discussed. In [22], the structure of the transformation matrix for the general bilinear transformation is examined. A new transformation matrix technique for bilinear transformation is proposed in [23], while in [24] properties of the known transformation matrix are presented. In [25]-[30], in order to achieve the desired transformation the method of the synthetic division is used. In [31], a closed form relationship for the multiple bilinear transformation is used by Erfani, Ahmadi, and Ramachandran.

In [32]-[36], one can find very important applications of several rational transformations. In [37], a very efficient algorithm based on the well-known Horner's form is proposed by Waggener. This is a simple recursive algorithm first for bilinear transformation and secondly for other single-variable rational transformations. Heinen and Siddique extended this technique for any arbitrary 1-D rational transformation [38]. One should note that several authors use the term "polynomial transformation" instead of the term rational transformation. In [40], fast multivariable bilinear and Hadamard transforms are presented.

In [41], an algorithm for any (2-D) spectral transformation of 2-D filters [1]-[3], [39], which is based on the technique of [37] and [38], is presented.

In the present paper, a new algorithm for the same problem is proposed. The algorithm is based on the discrete Fourier transform (DFT). The exploitation of the DFT in similar problems of systems theory is already known. In [42], the DFT is used in order to determine the characteristic polynomial of a rectangular matrix and in [43] the same technique is used for the calculation of a determinantal polynomial. The extension of this technique in 2-D systems is given in [44]. The use of DFT for arbitrary transformations of one-variable polynomials and ra- 
tional functions is known [45]. So, in the present paper the use of DFT is proposed in order to find a new efficient algorithm for spectral transformations for 2-D filters. The original polynomial under the transformation $T\left(s_{1}=G_{1}\left(z_{1}, z_{2}\right), s_{2}=G_{2}\left(z_{1}, z_{2}\right)\right)$, $G_{1}$ and $G_{2}$ being rational functions in $\left(z_{1}, z_{2}\right)$, is transformed to the rational function $\left(A\left(z_{1}, z_{2}\right) / B\left(z_{1}, z_{2}\right)\right)$. In Section II, the Horner's formula for a 2-D polynomial is described. In Section III, the algorithm is fully presented. In Section IV, the computational complexity of the presented algorithms is evaluated. Finally, Section V contains some concluding remarks.

\section{THE HORNER's FORM FOR 2-D POLYNOMIALS}

In what follows, it is necessary to describe the Horner's formula for 2-D Polynomials [41]. To this end, consider the polynomial $F\left(s_{1}, s_{2}\right)=\sum_{i_{1}=0}^{N_{1}} \sum_{i_{2}=0}^{N_{2}} f\left(i_{1}, i_{2}\right) s_{1}^{i_{1}} s_{2}^{i_{2}}$. This polynomial can be evaluated using the following 2-D Horner's form

$$
\begin{array}{r}
F^{(k, l)}\left(s_{1}\right)=F^{(k-1, l)}\left(s_{1}\right) \cdot s_{1}+f\left(N_{1}-k, N_{2}-l\right), \\
k=1, \ldots, N_{1}
\end{array}
$$

with the initial condition $F^{(0, l)}\left(s_{1}\right)=f\left(N_{1}, N_{2}-l\right)$ and

$$
\begin{array}{r}
\hat{F}^{(l)}\left(s_{1}, s_{2}\right)=\hat{F}^{(l-1)}\left(s_{1}, s_{2}\right) \cdot s_{2}+F^{\left(N_{1}, l\right)}\left(s_{1}, s_{2}\right), \\
l=1, \ldots, N_{2}
\end{array}
$$

with the initial condition $\hat{F}^{(0)}\left(s_{1}, s_{2}\right)=F^{\left(N_{1}, 0\right)}\left(s_{1}, s_{2}\right)$. In (2), $k$ is the step of the iteration and $l$ is simply a parameter $\left(l=0,1, \ldots, N_{2}\right)$ while in (3), $l$ is the step of the iteration. Finally, $F\left(s_{1}, s_{2}\right)=\hat{F}^{\left(N_{2}\right)}\left(s_{1}, s_{2}\right)$.

\section{SPeCtral TRANSFORMATIONS FOR 2-D FILTERS}

Consider the 2-D polynomial

$$
F\left(s_{1}, s_{2}\right)=\sum_{i_{1}=0}^{N_{1}} \sum_{i_{2}=0}^{N_{2}} f\left(i_{1}, i_{2}\right) s_{1}^{i_{1}} s_{2}^{i_{2}}
$$

Under the transformation

$$
\begin{aligned}
& s_{1}=G_{1}\left(z_{1}, z_{2}\right)=\frac{P_{1}\left(z_{1}, z_{2}\right)}{R_{1}\left(z_{1}, z_{2}\right)} \\
& s_{2}=G_{2}\left(z_{1}, z_{2}\right)=\frac{P_{2}\left(z_{1}, z_{2}\right)}{R_{2}\left(z_{1}, z_{2}\right)}
\end{aligned}
$$

where

$$
P_{j}\left(z_{1}, z_{2}\right)=\sum_{i_{1}=0}^{M_{1}} \sum_{i_{2}=0}^{M_{2}} p_{j}\left(i_{1}, i_{2}\right) z_{1}^{i_{1}} z_{2}^{i_{2}}, \quad j=1,2
$$

and

$$
R_{j}\left(z_{1}, z_{2}\right)=\sum_{i_{1}=0}^{M_{1}} \sum_{i_{2}=0}^{M_{2}} r_{j}\left(i_{1}, i_{2}\right) z_{1}^{i_{1}} z_{2}^{i_{2}}, \quad j=1,2
$$

where $F=F\left(s_{1}, s_{2}\right)$ is transformed to the rational function $\left(A\left(z_{1}, z_{2}\right) / B\left(z_{1}, z_{2}\right)\right)$. Suppose that

$$
\begin{aligned}
& A\left(z_{1}, z_{2}\right)=\sum_{i_{1}=0}^{\left(N_{1}+N_{2}\right) M_{1}} \sum_{i_{2}=0}^{\left(N_{1}+N_{2}\right) M_{2}} a\left(i_{1}, i_{2}\right) z_{1}^{i_{1}} z_{2}^{i_{2}} \\
& B\left(z_{1}, z_{2}\right)=\sum_{i_{1}=0}^{\left(N_{1}+N_{2}\right) M_{1}} \sum_{i_{2}=0}^{\left(N_{1}+N_{2}\right) M_{2}} b\left(i_{1}, i_{2}\right) z_{1}^{i_{1}} z_{2}^{i_{2}} .
\end{aligned}
$$

It is easy to verify that $B\left(z_{1}, z_{2}\right)=R_{1}^{N_{1}}\left(z_{1}, z_{2}\right) R_{2}^{N_{2}}\left(z_{1}, z_{2}\right)$.

In order to compute $A\left(z_{1}, z_{2}\right), B\left(z_{1}, z_{2}\right)$, we consider a 2-D discrete Fourier transform at $\left(r_{1}+1\right) \times\left(r_{2}+1\right)$ equispaced points on the unit 2-D disk where $r_{1}=\left(N_{1}+N_{2}\right) M_{1}, r_{2}=$ $\left(N_{1}+N_{2}\right) M_{2}$. So, we define

$$
w_{1}=e^{j 2 \pi /\left(r_{1}+1\right)}
$$

and

$$
w_{2}=e^{j 2 \pi /\left(r_{2}+1\right)} .
$$

The 2-D DFT for the double sequences of the coefficients $a\left(i_{1}, i_{2}\right)$ and $b\left(i_{1}, i_{2}\right)$, in (9) and (10), where $i_{1}=0, \ldots, r_{1}, i_{2}=0, \ldots, r_{2}$ is defined as follows:

$$
\begin{aligned}
& \tilde{b}\left(k_{1}, k_{2}\right)=\sum_{i_{1}=0}^{r_{1}} \sum_{i_{2}=0}^{r_{2}} b\left(i_{1}, i_{2}\right) w_{1}^{i_{1} k_{1}} w_{2}^{i_{2} k_{2}} \\
& \tilde{a}\left(k_{1}, k_{2}\right)=\sum_{i_{1}=0}^{r_{1}} \sum_{i_{2}=0}^{r_{2}} a\left(i_{1}, i_{2}\right) w_{1}^{i_{1} k_{1}} w_{2}^{i_{2} k_{2}} .
\end{aligned}
$$

So, $\tilde{b}\left(k_{1}, k_{2}\right), \tilde{a}\left(k_{1}, k_{2}\right)$ can be considered as the polynomial values of $B\left(z_{1}, z_{2}\right)$ and $A\left(z_{1}, z_{2}\right)$ at the equally spaced points $z_{1}=w_{1}^{k_{1}}, z_{2}=w_{2}^{k_{2}}$ on the unit 2-D disc, where $k_{1}=0, \ldots, r_{1}, k_{2}=0, \ldots, r_{2}$. So, the polynomial values $\tilde{b}\left(k_{1}, k_{2}\right)$ are found as follows:

$$
\tilde{b}\left(k_{1}, k_{2}\right)=\left.R_{1}^{N_{1}}\left(z_{1}, z_{2}\right) R_{2}^{N_{2}}\left(z_{1}, z_{2}\right)\right|_{z_{1}=w_{1}^{k_{1}}, z_{2}=w_{2}^{k_{2}}}
$$

while the coefficients $b\left(i_{1}, i_{2}\right)$ are evaluated via the inverse 2-D FFT as follows:

$$
b\left(i_{1}, i_{2}\right)=\frac{1}{r_{1}+1} \cdot \frac{1}{r_{2}+1} \sum_{k_{1}=0}^{r_{1}} \sum_{k_{2}=0}^{r_{2}} \tilde{b}\left(k_{1}, k_{2}\right) w_{1}^{-i_{1} k_{1}} w_{2}^{-i_{2} k_{2}} .
$$

Therefore, the polynomial $B\left(z_{1}, z_{2}\right)$ has been computed. Furthermore, the polynomial values $\tilde{a}\left(k_{1}, k_{2}\right)$ are found by the formula

$$
\begin{aligned}
\tilde{a}\left(k_{1}, k_{2}\right)= & \left.B\left(z_{1}, z_{2}\right)\right|_{z_{1}=w_{1}^{k_{1}}, z_{2}=w_{2}^{k_{2}}} \cdot \sum_{i_{1}=0}^{r_{1}} \sum_{i_{2}=0}^{r_{2}} f\left(i_{1}, i_{2}\right) \\
& \times\left.\left(\frac{P_{1}\left(z_{1}, z_{2}\right)}{R_{1}\left(z_{1}, z_{2}\right)}\right)^{i_{1}}\left(\frac{P_{2}\left(z_{1}, z_{2}\right)}{R_{2}\left(z_{1}, z_{2}\right)}\right)^{i_{2}}\right|_{z_{1}=w_{1}^{k_{1}}, z_{2}=w_{2}^{k_{2}}}
\end{aligned}
$$

or equivalently

$$
\begin{aligned}
& \tilde{a}\left(k_{1}, k_{2}\right)=\tilde{b}\left(k_{1}, k_{2}\right) \cdot \sum_{i_{1}=0}^{r_{1}} \sum_{i_{2}=0}^{r_{2}} f\left(i_{1}, i_{2}\right) \\
& \quad \times\left.\left(\frac{P_{1}\left(z_{1}, z_{2}\right)}{R_{1}\left(z_{1}, z_{2}\right)}\right)^{i_{1}}\left(\frac{P_{2}\left(z_{1}, z_{2}\right)}{R_{2}\left(z_{1}, z_{2}\right)}\right)^{i_{2}}\right|_{z_{1}=w_{1}^{k_{1}}, z_{2}=w_{2}^{k_{2}}}
\end{aligned}
$$

where Horner's formula for 2-D polynomials should be used.

Using, now, an inverse 2-D FFT, the coefficients $a\left(i_{1}, i_{2}\right)$ are evaluated as follows:

$$
a\left(i_{1}, i_{2}\right)=\frac{1}{r_{1}+1} \cdot \frac{1}{r_{2}+1} \sum_{k_{1}=0}^{r_{1}} \sum_{k_{2}=0}^{r_{2}} \tilde{a}\left(k_{1}, k_{2}\right) w_{1}^{-i_{1} k_{1}} w_{2}^{-i_{2} k_{2}}
$$

Therefore, the polynomial $A\left(z_{1}, z_{2}\right)$ has been found. 
Remark: In order to transform a given rational function $G\left(s_{1}, s_{2}\right)=\left(Q\left(s_{1}, s_{2}\right) / F\left(s_{1}, s_{2}\right)\right)$ under the transformation of (5) and (6), the previously developed procedure is applied for $Q\left(s_{1}, s_{2}\right)$ and $F\left(s_{1}, s_{2}\right)$ separately. Both these polynomials are transformed using the above algorithm where we are not interested in the common resultant denominator $B\left(z_{1}, z_{2}\right)$, but only for the different resultant numerators $A_{1}\left(z_{1}, z_{2}\right)$ and $A_{2}\left(z_{1}, z_{2}\right)$.

Example: Consider the 2-D digital filter with transfer function [2], [39] and [41]:

$$
\begin{aligned}
H\left(Z_{1}, Z_{2}\right) & =\frac{F_{1}\left(Z_{1}, Z_{2}\right)}{F_{2}\left(Z_{1}, Z_{2}\right)} \\
& =0.59944 \frac{\left[\begin{array}{ll}
1 & Z_{1}
\end{array}\right]\left[\begin{array}{cc}
1 & -0.04668 \\
-0.04668 & -0.46761
\end{array}\right]\left[\begin{array}{c}
1 \\
Z_{2}
\end{array}\right]}{\left[\begin{array}{ll}
1 & Z_{1}
\end{array}\right]\left[\begin{array}{cc}
1 & 0.42602 \\
0.42602 & -0.10692
\end{array}\right]\left[\begin{array}{c}
1 \\
Z_{2}
\end{array}\right]} .
\end{aligned}
$$

Under the spectral (rational) transformation

$$
\begin{aligned}
& Z_{1}=\frac{-0.2612+0.2612 z_{1}+0.2612 z_{2}+z_{1} z_{2}}{1+0.2612 z_{1}+0.2612 z_{2}-0.2612 z_{1} z_{2}} \\
& Z_{2}=\frac{0.7154-0.7154 z_{1}-0.7154 z_{2}+z_{1} z_{2}}{1-0.7154 z_{1}-0.7154 z_{2}+0.7154 z_{1} z_{2}}
\end{aligned}
$$

the above transfer function is transformed into another transfer function. First, we apply the whole procedure described above for the polynomial of the numerator of $H\left(Z_{1}, Z_{2}\right)$ for $z_{1}=$ $e^{j 2 \pi(n / 3)}, z_{2}=e^{j 2 \pi(m / 3)}, n=0,1,2$ and $m=0,1,2$. So, we find the following values that form a double sequence

$\{\{0.157584,0.459614+0.116335 j, 0.459614-0.116335 j\}$

$\{0.459614+0.116335 j, 3.29008-1.52751 j, 0.509692\}$

$\{0.459614-0.116335 j, 0.509692,3.29008+1.52751 j\}\}$.

Applying (double) inverse DFT, we find the double sequence

$$
\begin{aligned}
& \{\{1.06618,-0.625201,-0.0820382\} \\
& \{-0.625201,0.0018205,0.58987\} \\
& \{-0.0820382,0.58987,-0.675675\}\}
\end{aligned}
$$

which corresponds to the polynomial of the numerator of the new transfer function. We then apply the same procedure for the polynomial of the denominator of $H\left(Z_{1}, Z_{2}\right)$ for $z_{1}=e^{j 2 \pi(n / 3)}, z_{2}=e^{j 2 \pi(m / 3)}, n=0,1,2$ and $m=0,1,2$. So, we find again the following values:

$\{\{0.626389,0.225212+0.298092 j, 0.225212-0.298092 j\}$

$\{0.225212+0.298092 j, 2.67103-0.8238 j, 2.026\}$

$\{0.225212-0.298092 j, 2.026,2.67103+0.8238 j\}\}$.
Applying (double) inverse DFT we find the double sequence

$$
\begin{aligned}
& \{\{1.21348,-0.528443,-0.326097\} \\
& \{-0.528443,0.446268,0.388004\} \\
& \{-0.326097,0.388004,-0.100284\}\}
\end{aligned}
$$

which corresponds to the polynomial of the denominator of the new transfer function.

So, the resultant digital filter is described by the transfer function shown at the bottom of the page. This is a typical transformation of a high-pass 2-D digital filter [2].

\section{COMPUTATIONAL COMPLEXITY}

\section{A. For Finding $B\left(z_{1}, z_{2}\right)$}

Using the 2-D Horner's formula, for finding $R_{1}\left(z_{1}, z_{2}\right), M_{1} M_{2}+M_{1}+M_{2}$ complex multiplications and $M_{1} M_{2}+M_{1}+M_{2}$ complex additions are needed. Also, for finding $R_{2}\left(z_{1}, z_{2}\right)$, we need $M_{1} M_{2}+M_{1}+M_{2}$ complex multiplications and complex additions. So, in (15.1) there exists a cost of $2\left(M_{1} M_{2}+M_{1}+M_{2}\right)+N_{1}+N_{2}-1$ complex multiplications as well as a cost of $2\left(M_{1} M_{2}+M_{1}+M_{2}\right)$ complex additions. Since $\left(r_{1}+1\right)\left(r_{2}+1\right)$ number of equation (15.1) are required, i.e., $\left(\left(N_{1}+N_{2}\right) M_{1}+1\right)\left(\left(N_{1}+N_{2}\right) M_{2}+1\right)$, the total cost is finally $\left(\left(N_{1}+N_{2}\right) M_{1}+1\right)\left(\left(N_{1}+N_{2}\right) M_{2}+1\right)\left(2\left(M_{1} M_{2}+\right.\right.$ $\left.\left.M_{1}+M_{2}\right)+N_{1}+N_{2}-1\right)$ complex multiplications as well as $2\left(\left(N_{1}+N_{2}\right) M_{1}+1\right)\left(\left(N_{1}+N_{2}\right) M_{2}+1\right)\left(M_{1} M_{2}+M_{1}+M_{2}\right)$ complex additions.

Equation (15.2) requires $(1 / 4)\left(r_{1}+1\right)\left(r_{2}+1\right) \log _{2}\left(r_{1}+\right.$ 1) $\log _{2}\left(r_{2}+1\right)$ complex multiplications, i.e., $(1 / 4)\left(\left(N_{1}+\right.\right.$ $\left.\left.N_{2}\right) M_{1}+1\right)\left(\left(N_{1}+N_{2}\right) M_{2}+1\right) \log _{2}\left(\left(N_{1}+N_{2}\right) M_{1}+\right.$ 1) $\log _{2}\left(\left(N_{1}+N_{2}\right) M_{2}+1\right)$.

\section{B. For Finding $A\left(z_{1}, z_{2}\right)$}

In (16.1), using the 2-D Horner's formula, for finding $P_{1}\left(z_{1}, z_{2}\right)$ we need $M_{1} M_{2}+M_{1}+M_{2}$ complex multiplications and $M_{1} M_{2}+M_{1}+M_{2}$ complex additions. Also, for finding $P_{2}\left(z_{1}, z_{2}\right)$ we need $M_{1} M_{2}+M_{1}+M_{2}$ complex multiplications and $M_{1} M_{2}+M_{1}+M_{2}$ complex additions. Note that $R_{1}\left(z_{1}, z_{2}\right)$ and $R_{2}\left(z_{1}, z_{2}\right)$ are already known from the computation in (15.1). Additionally in (16.1) we need 2 complex divisions. So, we need $2\left(M_{1} M_{2}+M_{1}+M_{2}\right)+2$ CMADs (complex multiplications and divisions). Applying the usual 2-D Horner's formula, one finds $\left(N_{1} N_{2}+N_{1}+N_{2}\right)+2\left(M_{1} M_{2}+M_{1}+M_{2}+1\right)+1$ CMAD's and $\left(N_{1} N_{2}+N_{1}+N_{2}\right)+2\left(M_{1} M_{2}+M_{1}+M_{2}\right)$ complex additions.

$$
H\left(z_{1}, z_{2}\right)=0.59944 \frac{\left[\begin{array}{lll}
1 & z_{1} & z_{1}^{2}
\end{array}\right]\left[\begin{array}{ccc}
1.06618 & -0.625201 & -0.0820382 \\
-0.625201 & 0.0018205 & 0.58987 \\
-0.0820382 & 0.58987 & -0.675675
\end{array}\right]\left[\begin{array}{l}
1 \\
z_{2} \\
z_{2}^{2}
\end{array}\right]}{\left[\begin{array}{lll}
1 & z_{1} & z_{1}^{2}
\end{array}\right]\left[\begin{array}{ccc}
1.21348 & -0.528443 & -0.326097 \\
-0.528443 & 0.446268 & 0.388004 \\
-0.326097 & 0.388004 & -0.100284
\end{array}\right]\left[\begin{array}{c}
1 \\
z_{2} \\
z_{2}^{2}
\end{array}\right]}
$$


Since $\left(r_{1}+1\right)\left(r_{2}+1\right)(16.1)$ are required, i.e., $\left(\left(N_{1}+N_{2}\right) M_{1}+1\right)\left(\left(N_{1}+N_{2}\right) M_{2}+1\right)$, the total cost is finally $\left(\left(N_{1}+N_{2}\right) M_{1}+1\right)\left(\left(N_{1}+N_{2}\right) M_{2}+1\right)\left(\left(N_{1} N_{2}+\right.\right.$ $\left.\left.N_{1}+N_{2}\right)+2\left(M_{1} M_{2}+M_{1}+M_{2}+1\right)+1\right)$ CMAD's as well as $\left(\left(N_{1}+N_{2}\right) M_{1}+1\right)\left(\left(N_{1}+N_{2}\right) M_{2}+1\right)\left(\left(N_{1} N_{2}+N_{1}+\right.\right.$ $\left.\left.N_{2}\right)+2\left(M_{1} M_{2}+M_{1}+M_{2}\right)\right)$ complex additions.

Equation (16.2) requires $(1 / 4)\left(r_{1}+1\right)\left(r_{2}+1\right) \log _{2}\left(r_{1}+\right.$ 1) $\log _{2}\left(r_{2}+1\right)$ complex multiplications, i.e., $(1 / 4)\left(\left(N_{1}+\right.\right.$ $\left.\left.N_{2}\right) M_{1}+1\right)\left(\left(N_{1}+N_{2}\right) M_{2}+1\right) \log _{2}\left(\left(N_{1}+N_{2}\right) M_{1}+\right.$ 1) $\log _{2}\left(\left(N_{1}+N_{2}\right) M_{2}+1\right)$.

The conclusion is that our method requires $\mathbf{O}\left(2\left(N_{1}+\right.\right.$ $\left.N_{2}\right)^{2} M_{1}^{2} M_{2}^{2}$ ) CMAD's with respect to $M_{1}, M_{2}$ (this is the evaluated complexity if $N_{1}, N_{2}$ are considered constant and $M_{1}, M_{2}$ variable) as well as $\mathbf{O}\left(\left(N_{1}+N_{2}\right)^{2} N_{1} N_{2} M_{1} M_{2}\right)$ CMAD's with respect to $N_{1}, N_{2}$ (this is the evaluated complexity if $M_{1}, M_{2}$ are considered constant and $N_{1}, N_{2}$ variable). Also our method requires $\mathbf{O}\left(\left(N_{1}+N_{2}\right)^{2} M_{1}^{2} M_{2}^{2}\right)$ complex additions with respect to $M_{1}, M_{2}$ as well as $\mathbf{O}\left(\left(N_{1}+N_{2}\right)^{2} N_{1} N_{2} M_{1} M_{2}\right)$ complex additions with respect to $N_{1}, N_{2}$. Note that each complex multiplication is implemented by four real multiplications and two real additions while each complex addition is implemented by two real additions.

So, the present method seems to be better than that of [41], where we had (1/10) $M_{1}^{2} M_{2}^{2} N_{2} \mathbf{O}\left(N_{1}^{5}\right)$ MAD's (and equal number of additions) with respect to $N_{1}$ and $(1.5 / 10) M_{1}^{2} M_{2}^{2} \mathbf{O}\left(N_{2}^{5}\right)$ MAD's (and equal number of additions) with respect to $N_{2}$ as well as $\mathbf{O}\left(M_{1}^{2} M_{2}^{2}\left[\left(N_{1}^{5} N_{2} / 10\right)+\right.\right.$ $\left(3 N_{1}^{4} N_{2} / 10\right)+\left(3 N_{1}^{3} N_{2}^{2} / 2\right)+\left(3 N_{1}^{2} N_{2}^{3} / 2\right)+\left(3 N_{1} N_{2}^{4} / 4\right)$ $\left.\left.+\left(3 N_{2}^{5} / 20\right)+\left(N_{1}^{4} / 4\right)+N_{1}^{3}+\left(3 N_{1}^{2} N_{2}^{2} / 2\right)+N_{1} N_{2}^{3}+\left(N_{2}^{4} / 4\right)\right]\right)$ with respect to $M_{1}, M_{2}$.

\section{CONCLUSION}

The algorithm presented in this paper speeds up the various 2-D rational transformations. These rational transformations are of great interest in the area of 2-D digital filters analysis and synthesis as well as in the area of 2-D signal processing. An important example is the double bilinear transformation via which a discrete 2-D system is transformed to a continuous one and vice-versa. The comparison of this method with a previously published one, [41], proves that the present method is better with respect to the computational complexity. Moreover, the whole formulation of the present method is simpler and more comprehensive than that of [41].

\section{REFERENCES}

[1] D. E. Dudgeon and R. M. Mersereau, Multidimensional Digital Signal Processing. Englewood Cliffs, NJ: Prentice-Hall, 1984.

[2] N. E. Pendegrass, S. K. Mitra, and E. I. Jury, "Spectral transformations for two-dimensional digital filters," IEEE Trans. Circuits Syst., vol. CAS-23, pp. 26-35, Jan. 1976.

[3] S. Chakrabarti and S. K. Mitra, "Design of two-dimensional digital filters via spectral transformations," Proc. IEEE, vol. 65, pp. 905-914, June 1977.

[4] C. J. Greaves, G. A. Gagne, and G. W. Bordner, "Evaluation of integrals appearing in minimization problems of discrete-data systems," IEEE Trans. Automat. Control, vol. AC-11, pp. 145-148, Jan. 1966.

[5] H. M. Power, "The mechanics of the bilinear transformation," IEEE Trans. Educ., vol. E-10, pp. 114-116, June 1967.

[6] - "Comments on the mechanics of the bilinear transformation," IEEE Trans. Educ., vol. E-11, p. 159, June 1968.

[7] D. C. Fielder, "Some classroom comments on bilinear transformations," IEEE Trans. Educ., vol. E-13, pp. 105-107, Aug. 1970.
[8] H. M. Power, "Further comments on the mechanics of the bilinear transformations," IEEE Trans. Educ., vol. E-13, pp. 114-115, Aug. 1970

[9] A. M. Bush and D. C. Fielder, "Simplified algebra for the bilinear and related transformations," IEEE Trans. Audio Electroacoust., vol. AU-21, pp. 127-128, Apr. 1973.

[10] N. E. Mastorakis, "Bilinear transformations of multidimensional systems: A tensor approach," Found. Comput., Decision Sci., vol. 20, no. 4, pp. 307-316, 1995

[11] E. I. Jury, "Remarks on the mechanics of the bilinear transformation," IEEE Trans. Audio Electroacoust., vol. AU-21, pp. 380-382, Aug. 1973.

[12] E. I. Jury and O. W. C. Chan, "Combinatorial rules for some helpful transformations," IEEE Trans. Circuit Theory, vol. CT-20, pp. 476-480, Sept. 1973.

[13] K. A. Moore, "An APL program for bilinear transformation," IEEE Trans. Acoust., Speech, Signal Processing, vol. ASSP-22, pp. 225-226, June 1974.

[14] N. K. Bose and E. I. Jury, "Positivity and stability tests for multidimensional filters (discrete-continuous)," IEEE Trans. Acoust., Speech, Signal Processing, vol. ASSP-22, pp. 174-180, June 1974.

[15] V. V. B. Rao and V. K. Aatre, "Transformation of rational functions," Proc. IEEE, pp. 1028-1029, July 1974.

[16] V. V. B. Rao and V. K. Aatre, "Transformation matrices for bilinear transformation of multivariable polynomials," IEEE Trans. Acoust., Speech, Signal Processing, vol. ASSP-24, pp. 266-267, June 1976.

[17] C. F. Chen and Y. T. Tsay, "A new formula for the discrete-time system stability test," Proc. IEEE, vol. 65, pp. 1200-1202, Aug. 1977.

[18] B. O'Connor and T. S. Huang, "An efficient algorithm for bilinear transformation of multivariable polynomials," IEEE Trans. Acoust., Speech, Signal Processing, vol. ASSP-26, pp. 380-381, Aug. 1978.

[19] S.-C. Pei, "Multidimensional bilinear transformations," Electron. Lett., vol. 18, no. 19, pp. 1069-1070, 1982.

[20] N. K. Bose, "Properties of the Qn-matrix in bilinear transformation," Proc. IEEE, vol. 71, pp. 1110-1111, Sept. 1983.

[21] D. Hertz and E. Zeheb, "A general property of the transformation matrices associated with the $n$-variable bilinear transformation," IEEE Trans. Circuits Syst., vol. CAS-31, pp. 296-299, Mar. 1984.

[22] N. M. Smart and S. Barnett, "Transformation matrices in the general bilinear transformation of multivariable polynomials," IEEE Trans. Circuits Syst., vol. ASSP-32, pp. 634-636, June 1984.

[23] V. V. B. Rao and K. S. Rao, "A new transformation matrix for bilinear transformation," IEEE Trans. Acoust., Speech, Signal Processing, vol. ASSP-32, pp. 933-934, Aug. 1984.

[24] V. V. B. Rao and K. N. Rao, "A note on transformation matrices for bilinear transformation," IEEE Trans. Circuits Syst., vol. CAS-32, pp. 857-858, Aug. 1985.

[25] A. C. Davies, "Bilinear transformation of polynomials," IEEE Trans. Circuits Syst., pp. 792-794, Nov. 1974.

[26] M. Policastro, "A Simple algorithm to perform the bilinear transformation," Int. J. Control, vol. 30, no. 4, pp. 713-715.

[27] R. Parthasarathy and K. N. Jayasimha, "Bilinear transformation by synthetic division," IEEE Trans. Automat. Control, vol. AC-29, pp. 575-576, June 1984.

[28] — "Bilinear transformation of multivariable polynomials," IEEE Trans. Acoust., Speech, Signal Processing, vol. ASSP-32, pp. 177-178, Feb. 1984.

[29] H. S. Malvar, "Comments on bilinear transformation by synthetic division," IEEE Trans. Automat. Control, vol. AC-30, p. 416, Apr. 1985.

[30] N. M. Smart and S. Barnett, "Bilinear transformation of multivariable polynomials using the Horner method," Int. J. Control, vol. 37, no. 4, pp. $861-865,1983$.

[31] S. Erfani, M. Ahmadi, and V. Ramachandran, "On the algebra of multiple bilinear transformations," J. Franklin Inst., vol. 322, no. 3, pp. $137-142,1986$.

[32] S. Barnett, "Some applications of matrices to location of zeros of polynomials," Int. J. Control, vol. 17, no. 4, pp. 823-831, 1973.

[33] E. I. Jury and T. Y. Ruridant, "On the evaluation of double square integral in the $\left(s_{1}, s_{2}\right)$ complex biplane," IEEE Trans. Acoust., Speech, Signal Processing, vol. ASSP-34, pp. 630-632, Mar. 1986.

[34] E. I. Jury and P. Bauer, "On the stability of two-dimensional continuous systems," IEEE Trans. Circuits Syst., vol. CAS-35, pp. 1487-1500, Dec. 1988

[35] A. Moshfegh, "Discrete state-space modeling for linear $n$ th-order constant coefficient distributed-parameter systems," Dyn. Control, vol. 3, no. 1 , pp. 71-90, 1993.

[36] N. E. Mastorakis, "A general factorization method for multivariable polynomials," Multidimensional Systems and Signal Processing, vol. 5, pp. 151-178, 1994. 
[37] W. N. Waggener, "Recursive algorithms for polynomial transformations," IEEE Circuits Syst. Mag., vol. 2, pp. 3-5, Sept. 1980

[38] J. A. Heinen and B. M. A. Siddique, "A simple algorithm for arbitrary polynomial transformation," IEEE Trans. Acoust., Speech, Signal Processing, vol. ASSP-36, pp. 77-80, Jan. 1988.

[39] G. A. Maria and M. M. Fahmy, "An $l_{p}$ design technique for two-dimensional digital recursive filters," IEEE Trans. Acoust., Speech, Signal Processing, vol. ASSP-22, pp. 15-21, Jan. 1974.

[40] D. Hertz, "On fast multivariable bilinear and hadamard transforms," IEEE Trans. Signal Processing, vol. 39, pp. 1788-1792, Aug. 1991.

[41] N. Mastorakis, "Recursive algorithms for two-dimensional filters' spectral transformations," IEEE Trans. Signal Processing, vol. 44, pp. 2647-2651, Oct. 1996.

[42] T. Lee, "A simple method to determine the characteristic function $f(\lambda)=|\lambda I-A|$ by discrete Fourier series and fast Fourier transform," IEEE Trans. Circuits Syst., vol. 23, p. 242, Apr. 1976.

[43] L. Paccagnella and G. Pierobon, "FFT calculation of a determinantal polynomial," IEEE Trans. Circuits Syst., vol. 21, pp. 401-402, June 1974.

[44] G. Antoniou, S. Mentzelopoulou, and G. Glentis, "Determination of transfer function of two-dimensional generalized systems using the discrete Fourier transform," in Inst. Elect. Eng. Proc. D, vol. 38, July 1991, pp. 327-330.

[45] S. C. Chan and K. L. Ho, "A new algorithm for arbitrary transformation of polynomial and rational functions," IEEE Trans. Signal Processing, vol. 40, pp. 456-460, Feb. 1992.

[46] D. T. Whiteside, Ed., The Mathematical Papers of Isaac Newton. Cambridge, MA: Cambridge Univ. Press, 1968.

[47] A. V. Aho, J. E. Hopcroft, and J. D. Ullman, The Design and Analysis of Computer Algorithms. Reading, MA: Addison Wesley, 1974.

[48] D. E. Knuth, The Art of Computer Programming, 2nd ed. Reading, MA: Addison Wesley, 1981, vol. 2.

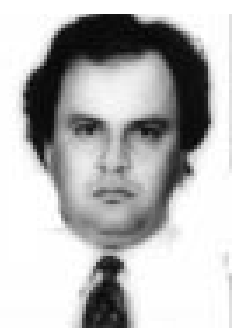

Nikos E. Mastorakis (M'97-SM'99) received the B.Sc. and M.Sc. degrees in electrical engineering, and the Ph.D. degree in electrical engineering and computer science from the National Technical University, Athens, Greece. He also received the B.Sc. (Ptychion) in pure mathematics from the same university.

He have served as special scientist on Computers and Electronics in the Hellenic (Greek) Army Genera Staff (1993-1994) and taught courses in the Electrical Engineering Department of the National Technical University of Athens (1998-1994). He has also served as Visiting Professor at the University of Exeter, School of Engineering (U.K., 1998) while he is currently Professor and Head of the Department of Computer Science at the Hellenic Naval Academy, Piraeus, Greece. He has published over than 200 papers in international books, journals and conferences and is active reviewer of more than 20 International Journals.

Dr. Mastorakis has received several awards (Royal Society of England, Hellenic National Research Foundation, etc.) for his academic studies and his scientific research. His biography is included in many international biographical reference publications.

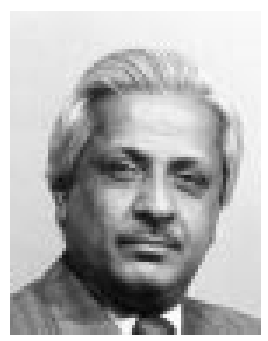

M. N. S. Swamy (S'59-M'62-SM'74-F'80-LF'01) received the B.Sc. (Hons.) degree in mathematics from Mysore University, India, in 1954, the Diploma in electrical communication engineering from the Indian Institute of Science, Bangalore in 1957, and the M.Sc. and Ph.D. degrees in electrical engineering from the University of Saskatchewan, Saskatoon, Canada, in 1960 and 1963, respectively. In August 2001 he was awarded a Doctor of Science in engineering (Honoris Causa) by Ansted University "In recognition of his exemplary contributions to the research in Electrical and Computer Engineering and to Engineering Education".

He is currently a Research Professor and the Director of the Center for Signal Processing and Communications in the Department of Electrical and Computer Engineering at Concordia University, Montreal, Canada, where he served as the Chair of the Department of Electrical Engineering from 1970 to 1977, and Dean of Engineering and Computer Science from 1977 to 1993. Since July 2001, he holds the Concordia Chair (Tier I) in Signal Processing. He has also taught in the Electrical Engineering Department of the Technical University of Nova Scotia, Halifax, and the University of Calgary, Calgary, as well as in the Department of Mathematics at the University of Saskatchewan. He has published extensively in the areas of number theory, circuits, systems and signal processing, and holds four patents. He is the co-author of two book chapters and three books: Graphs, Networks and Algorithms (New York, Wiley, 1981), Graphs: Theory and Algorithms (New York, Wiley, 1992), and Switched Capacitor Filters: Theory, Analysis and Design (Prentice Hall International UK Ltd., 1995). A Russian Translation of the first book was published by Mir Publishers, Moscow, in 1984, while a Chinese version was published by the Education Press, Beijing, in 1987. He is a member of Micronet, a National Network of Centers of Excellence in Canada, and also its coordinator for Concordia University.

Dr. Swamy is a Fellow of the Institute of Electrical Engineers (U.K.), the Engineering Institute of Canada, the Institution of Engineers (India), and the Institution of Electronic and Telecommunication Engineers (India). Presently, he is Vice-President (Publications) for the Circuits and Systems (CAS) Society. He has served the IEEE in various capacities such as the Vice President of the CAS society in 1976, Editor-in-Chief of the IEEE TRANSACTIONS ON CIRCUITS AND SYSTEMS I during 1999 to 2001, Associate Editor of the IEEE TRANSACTIONS ON CIRCUITS AND SYSTEMS during 1985 to 1987, Program Chair for the 1973 IEEE CAS Symposium, General Chair for the 1984 IEEE CAS Symposium, Vice-Chair for the 1999 IEEE CAS Symposium and a member of the Board of Governors of the CAS Society. He is the recipient of many IEEE-CAS Society awards including the Education Award in 2000, Golden Jubilee Medal in 2000, and the 1986 Guillemin-Cauer Best Paper Award. 